\title{
Cognitive-Behavioral Intervention for Distress in Patients with Melanoma
}

\section{Comparison with Standard Medical Care and Impact on Quality of Life}

\author{
Peter C. Trask, Ph.o. ${ }^{1}$ \\ Amber G. Paterson, Ph.D. ${ }^{2}$ \\ Kent A. Griffith, M.S., M.P.H. ${ }^{3}$ \\ Michelle B. Riba, M.D. ${ }^{4}$ \\ Jennifer L. Schwartz, M.D. ${ }^{5}$ \\ ${ }^{1}$ Behavioral Medicine Program, University of \\ Michigan, Ann Arbor, Michigan. \\ ${ }^{2}$ Kaplan and Kaplan Psychologists, Ontario, Can- \\ ada. \\ ${ }^{3}$ Biostatistics Unit, University of Michigan Compre- \\ hensive Cancer Center, Ann Arbor, Michigan. \\ ${ }^{4}$ Department of Psychiatry, University of Michigan, \\ Ann Arbor Michigan. \\ ${ }^{5}$ Department of Dermatology, University of Mich- \\ igan, Ann Arbor, Michigan.
}

Supported by the University of Michigan Faculty Group Practice (VIF Number 99-101) and by National Institutes of Health grant P30 CA46592.

The authors thank Dr. Timothy Johnson for his support of the project.

Address for reprints: Peter C. Trask, Ph.D., Behavioral Medicine Program, University of Michigan, 475 Market Place, Suite L, Box 7, Ann Arbor, Ml 48108-0757; Fax: (734) 998-6423; E-mail: pctrask@umich.edu

Received December 31, 2002; revision received April 23, 2003; accepted May 12, 2003.
BACKGROUND. Melanoma accounts for $>79 \%$ of skin cancer-related deaths, although it accounts for only $4 \%$ of skin cancer incidence. Given the potential for lethality, it is likely that patients with melanoma may experience significant emotional distress. The current study was designed to determine the effect of a cognitive-behavioral intervention on distress and health-related quality of life (HRQOL) in patients with melanoma who had medium-to-high distress.

METHODS. Forty-eight patients who had Global Severity Index scores $\geq 602$ months after their initial visit to the multidisciplinary melanoma clinic were randomized to receive either standard care or 4 sessions of a cognitive-behavioral intervention (CBI). Repeated assessments using the Brief Symptom Inventory, the Medical Outcomes Survey Short Form-36, and the State-Trait Anxiety Inventory occurred at baseline, at 2 months, and at 6 months after intervention for both groups.

RESULTS. An intent-to-treat analysis did not reveal significantly lower distress in the CBI group at 2 months or 6 months of follow-up, although differences were noted in anxiety and HRQOL. An effect-of-intervention analysis did reveal lower levels of distress in the CBI group at 2 months, with differences approaching significance at 6 months.

CONCLUSIONS. The four-session CBI significantly reduced distress and improved HRQOL for a period of 2 months in patients with melanoma who had mediumto-high distress, with improved general health evident 6 months after the intervention. Some variation in results was revealed in an intent-to-treat analysis. The initial evidence from the current study showed that a brief intervention may be effective for creating change in individuals with cancer who have increased distress, although further research is needed to identify the most optimal approach for delivering the intervention. Cancer 2003;98:854-64.

(C) 2003 American Cancer Society.

KEYWORDS: intent-to-treat analysis, melanoma, distress, cognitive-behavioral interventions.

M elanoma is the seventh most common cancer in the United States and is the fifth most common cancer in men. It also is the second most common cancer in women ages 30-35 years and the most common cancer in women ages $25-29$ years. ${ }^{1,2}$ The incidence of melanoma is greater in men compared with women, ${ }^{2,3}$ with $>25 \%$ of melanomas occurring in individuals younger than age 45 years. Since 1973 , the incidence rate per year per 100,000 population has more than doubled from 6.8 per 100,000 population to 17.4 per 100,000 population. ${ }^{3}$ Although it accounts for only $4 \%$ of diagnoses of skin cancer, melanoma accounts for $>79 \%$ of skin cancer-related deaths. 
Current projections indicate that approximately 53,600 Americans are expected to be diagnosed with melanoma in the year $2002 .{ }^{4}$ The rising incidence rate for melanoma is of concern, because it is a particularly deadly form of skin cancer if it is allowed to advance. In particular, 5-year survival rates are $>90 \%$ in patients with melanoma classified as Stage I (tumor thickness $\leq 1.0 \mathrm{~mm}$ or tumor thickness $1.01-2.0 \mathrm{~mm}$ without ulceration). ${ }^{5}$ Similar to with other types of cancers, survival decreases dramatically as the tumor advances, with 5-year survival rates in patients with Stage IV melanoma (distant metastases) approaching $10 \%$. Given the rapidly increasing incidence and potential for lethality of this disease, it would not be surprising to find that distress is as prevalent in these patients as it is in patients with other types of malignant disease.

Indeed, in a previous report, ${ }^{6}$ we identified significant levels of emotional distress (i.e., increased levels of anxiety, depression, and adjustment disorders) in approximately $30 \%$ of patients melanoma, a level that matches the level of distress identified in more general samples of patients with cancer. ${ }^{7,8}$ Distress has been associated with decreased quality of life (QOL), increased recurrence and mortality rates, increased costs and utilization of resources, and decreased adherence to treatment, including chemotherapy. ${ }^{9-11}$ Early identification of distress and institution of effective treatment to relieve it may provide benefits to patients, providers, and the health care systems in which they participate. One way that it may provide benefits to patients is through improved QOL. QOL has been an important research outcome measure in patients with cancer for the past 15 years, although it rarely is assessed or included as an objective in clinical oncology research trials. ${ }^{12}$ Although it is somewhat difficult to define, Cella and Bonomi ${ }^{13}$ have identified health-related QOL as the degree to which a medical diagnosis or treatment for a patient with such a diagnosis affects an individual's physical, social, and emotional well-being. Several measures have been developed to assess the impact of medical illness on well being, including the Medical Outcomes Survey-Short Form-36 (SF-36).

The vast majority of research into cancer-related QOL has focused on patients with either breast carcinoma or prostate carcinoma. In these populations, QOL has been related significantly to distress and symptom presence (e.g., fatigue, pain, nausea). ${ }^{14,15}$ Few studies, however, have researched the QOL of patients with melanoma. An exception is a recent study by Brown et al., ${ }^{16}$ who examined the course of QOL in patients with late-stage melanoma (Stage IV) every 3 months for up to 2 years and found consider- able variability between patients. Despite this finding, on average, a reduction in physical well-being was noted, which was not surprising given the poor prognosis for these patients. To our knowledge, this is the only study documenting the course of QOL in patients with melanoma. Thus, QOL has not been examined in patients with earlier stage melanoma, and no studies have focused on testing an intervention designed to improve the QOL of patients with melanoma.

Currently, significant numbers of studies have been designed to treat distress in patients with cancer (for reviews, see Trijsburg et al. ${ }^{17}$ and Andersen ${ }^{18}$ ). Interventions have ranged from individuals to groups, have included educational as well as therapeutic information, and have been delivered in several different modalities with positive outcomes. ${ }^{18}$ Studies suggest that regardless of the method of delivery (e.g., face-to-face, telephone), the most effective interventions for treating emotional distress focus on providing education, behavioral training, stress management, cognitive therapy, and support. ${ }^{19-22}$ Previous results from group intervention studies for reducing distress in patients with melanoma have reported positive improvements in distress, coping, and survival. ${ }^{9}$ Many of these interventions, however, are less structured and longer-term; as such, they may not fit easily into existing medical systems. Few brief, replicable therapeutic interventions aimed at decreasing distress and improving QOL for specific subpopulations of patients with cancer have been tested. Thus, the current study was designed to determine the effect of a brief, manualized, cognitive-behavioral intervention (CBI) compared with standard medical care on distress and QOL in patients with medium and high distress. It was hypothesized that patients who received the intervention would report significantly lower levels of distress and improved QOL compared with preintervention scores and compared with a group of patients who received standard medical care.

\section{MATERIALS AND METHODS \\ Patients}

This study was part of a larger study of distress in patients with melanoma conducted in the Multidisciplinary Melanoma Clinic (MMC) at the University of Michigan's Comprehensive Cancer Center. ${ }^{6}$ Participants for the study were recruited after approval of the study was obtained from the University of Michigan's Institutional Review Board. In the larger study, patients were referred to the MMC by primary care physicians, dermatologists, internists, and surgeons; and they were eligible for participation if they were older than age 18 years, able to read and understand English, and able to give their own consent, and if they 
did not have a history of chemical dependency, inpatient psychiatric treatment, head injury with loss of consciousness, or Stage IV melanoma. Three hundred seventy-five patients met the inclusion criteria and provided information from their initial visit to the MMC.

An additional criterion for this part of the study was a 2-month assessment Brief Symptom Inventory (BSI) Global Severity Index (GSI) score $>60$. Of 247 patients who completed the 2-month assessment, 52 patients met this criterion. The average age of participants was 53.4 years (standard deviation [SD], 15.36 years; range, 22-92 years). Thirty-eight patients (73\%) were married, with the remaining 14 patients $(27 \%)$ divorced, separated, widowed, or never married. The sample was highly educated, with $69 \%$ of patients having completed at least 1 year of college (mean $\pm \mathrm{SD}, 4.86 \pm 2.72$ years of college). Sixteen patients were male. Twenty-nine patients $(50 \%)$ were working, 15 patients $(29 \%)$ were retired, and the remaining 8 patients $(15 \%)$ were not working. Patients had their disease staged according to the American Joint Committee on Cancer (AJCC) criteria; $26 \%$ of patients had Stage 0 disease, $44 \%$ of patients had Stage I disease, $22 \%$ of patients had Stage II disease, and $8 \%$ of patient had Stage III disease.

\section{Procedure}

Participants completed the BSI, the SF-36, and the State-Trait Anxiety Inventory (STAI) at their initial presentation to the MMC and again 2 months later after they underwent any initial surgical procedure. For the purposes of the current study, this second assessment served, and is referred to, as the baseline assessment (t0). Participants who met the aforementioned criteria at this assessment were assigned randomly to receive either standard medical treatment or standard medical treatment plus CBI.

Individuals who were assigned randomly to the CBI treatment group received a psychiatric intake and 3 subsequent 50-minute weekly sessions focused on relaxation training, cognitive challenging, and problem solving. Each session was devoted to a discussion of one of the topics, with the content tailored to specific patient examples. Patients attended sessions once per week over a period of 4 weeks. The average interval between the baseline assessment ( $\mathrm{t} 0$ ) and the second assessment (t2) assessment was 3.31 months (SD, 0.73 months). Two months (t2) and 6 months (t6) after the last psychotherapy session, individuals in the interventional and control groups were sent additional questionnaire packages to complete.

\section{Measures \\ BSI}

The BSI is a 53-item measure of emotional distress that takes 5-10 minutes to complete. ${ }^{23,24}$ It is the short version of the revised Symptom Check List and has well-demonstrated reliability and validity. ${ }^{25}$ Individual items are answered on a $0-4$ scale $(0=$ not at all distressed; 4 = extremely distressed) and are summed into 1 of 9 clinical scales and 3 summary scales. Specific test-retest reliability over a 2 -week interval ranges from 0.68 for the Somatization scale to 0.91 for Phobic Anxiety. The GSI, which provides the most sensitive measure of overall distress, has a stability coefficient of 0.90. Thus, the BSI, and the GSI scale in particular, have excellent ability to consistently measure distress over time. ${ }^{23,24}$ The BSI is standardized using area Tscores, each with a mean of 50 and an SD of 10. In other studies with patients who had cancer, the GSI scale of the BSI was sensitive for detecting changes after psychologic interventions. ${ }^{22}$

\section{SF-36}

The SF-36 is a 36-item questionnaire that assesses health functioning. ${ }^{26}$ It is composed of eight scales that include physical functioning, social functioning, bodily pain, and mental health. It has demonstrated reliability and validity. In particular, 2-week and 6-month test-retest reliability for the scales ranges from 0.43 to 0.90 , and the majority of scales have reliability scores $>0.60$, demonstrating their use as a measure of health over time. ${ }^{26}$ The SF-36 was chosen over several more specific measures of QOL (e.g., the Functional Assessment of Cancer Treatment or the Functional Living Index for Cancer) because of the desire to obtain a measure of initial health functioning when individuals may be unaware of their diagnosis of cancer. Because the SF-36 does not specifically mention cancer, individuals are not likely to become more distressed by completing the questionnaire. It was used in the current study as a general measure of QOL.

\section{STAI}

The STAI is a 40 -item questionnaire that was designed to assess transient or situational and stable or dispositional symptoms of anxiety. ${ }^{27,28}$ The questionnaire consists of 2 series of 20 statements that have been used to describe individuals (e.g., I feel nervous and restless). Participants are asked to respond to the first 20 statements (assessing state anxiety) according to how well each describes them at the moment they complete the questionnaires and to the second 20 statements (assessing trait anxiety) according to how they generally feel. Responses are rated from 1 (not at 
all/almost never) to 4 (very much so/almost always). Scores are summed and divided by 20 to provide average state and trait anxiety scores. Test-retest stability coefficients are reasonably high for the trait scale and low for the state scale, as expected given the two aspects of anxiety measured. ${ }^{28}$ The STAI was chosen to provide an additional measure of anxiety and because of its ability to differentiate between situational and more characteristic manifestations of anxiety.

\section{Statistical Analyses}

The a priori primary objectives of the current study were as follows: first, to identify levels of distress, health functioning, and coping styles of individuals upon initial presentation for the treatment of melanoma at a large tertiary-care clinic; and, second, to identify patients who were experiencing high emotional distress after undergoing surgical treatment for melanoma and to determine the influence of providing a CBI, compared with standard medical care, on emotional distress. Secondary objectives included 1) reporting the differences in subjective QOL scores (SF36) between patients who received the CBI and patients who received standard medical care and 2) describing the longitudinal course of distress and health functioning. For the first primary objective, a crosssectional description of the level of distress, QOL, and coping skills of patients with melanoma have been published previously. ${ }^{6}$ The current report includes the results of the latter primary and secondary objectives. The study was designed specifically to identify at least 50 patients who reported moderate distress (GSI $\geq 60$ ) at their postsurgical follow-up visit and to randomized those patients evenly to receive either the CBI or standard medical care.

Historical information from a previously conducted study that examined pretherapeutic and posttherapeutic interventions for emotional distress, as measured by the GSI of the BSI, was used to estimate the mean and variability of GSI scores for this trial. Specifically, the mean GSI score at baseline was estimated at approximately 64.0, with an SD of 8.2 and a correlation between preintervention and postintervention scores of 0.6. Assuming constant variance over time, it was estimated that with 50 patients randomized evenly to receive either intervention or observation, the trial would have at least $90 \%$ power to detect a difference in distress of at least 10 points between groups. Twenty-five patients per group was the chosen sample size to allow for high power for the group comparison, even if the drop-out rate approached $20 \%$ for the trial as a whole.

Patients were randomized to receive usual care or CBI in a one-to-one fashion using a blinded, block- randomization scheme. Although they agreed to the conditions of randomization, several participants who were randomized to receive the CBI either were unable to attend or chose not to attend sessions. Consequently, three groups emerged: Patients who were randomized to received and received the intervention, patients who were randomized to receive and chose not to receive the intervention, and patients who were randomized to receive standard medical care. Given these groups, two separate analytic strategies were employed: one based on randomization, an intent-totreat (ITT) analysis, and the other based on whether the patient received the CBI, an effect-of-intervention (EOI) analysis. Baseline demographic features for the patients were compared by randomization (ITT) or by intervention status (EOI) using the Fisher exact test for characteristics that were categoric in nature and the two-sample $t$ test for continuously distributed characteristics. Not all patients responded to questionnaires at each time point (sample sizes for each group at each survey time point are listed in Tables 1 and 2); therefore, longitudinal changes in distress, as measured by the GSI, other BSI subscales, and the subscales of the SF-36, were compared over time using mixed-effects, repeated-measures models. $^{29}$ The mixed-effects model allowed the modeling of all available survey information at each time point, with the implicit assumption that missingness is an ignorable random process, often called missing at random. Each patient's longitudinal time course for the outcome of interest (e.g., GSI, SF-36 subscale, etc.) was modeled allowing for random deviations from the group mean at baseline (intercept) and over time (slope). It was assumed that correlations within a category over time followed an unstructured form, rather than an autoregressive form, because the latter may be influenced unduly by missing data. ${ }^{29}$ Randomization assignment and intervention status were modeled as fixed effects. Fixed-effect group means were modeled as a function of time and time squared to allow for curvature in the average profile over time ${ }^{30,31}$ Estimated group means were compared using appropriate contrasts with the calculation of the F-statistic at each survey point. Fstatistics, given the numerator and denominator degrees of freedom, with $P$ values less than the standard, acceptable type I error rate of $5 \%$ were considered significantly different.

\section{RESULTS}

Fifty-two patients met the criteria for medium-to-high distress (GSI score $\geq 60$ ) at postsurgical follow-up, although 4 patients declined further participation and were not randomized to intervention conditions. Therefore, for the ITT analysis, 25 individuals were 
TABLE 1

Patient Characteristics: Intent-to-Treat Comparison

\begin{tabular}{|c|c|c|}
\hline Characteristic & $\begin{array}{l}\text { Randomized to } \\
\text { intervention }\end{array}$ & $\begin{array}{l}\text { Randomized to } \\
\text { control }\end{array}$ \\
\hline \multicolumn{3}{|l|}{ Sample size } \\
\hline Baseline & 25 & 23 \\
\hline Two months & 21 & 18 \\
\hline Six months & 18 & 16 \\
\hline \multicolumn{3}{|l|}{ Male gender } \\
\hline No. & 8 & 6 \\
\hline$\%$ at Month 2 & 32.0 & 26.1 \\
\hline \multicolumn{3}{|l|}{ Age (yrs) } \\
\hline Mean & 56.2 & 51.0 \\
\hline Range & $30-92$ & $22-71$ \\
\hline \multicolumn{3}{|l|}{ Education (yrs) } \\
\hline Mean & 14.6 & 14.9 \\
\hline Range & $12-23$ & $12-21$ \\
\hline \multicolumn{3}{|l|}{ No. of children } \\
\hline Median & 2.1 & 2.3 \\
\hline Range & $0-5$ & $0-5$ \\
\hline \multicolumn{3}{|l|}{ Martial status (\%) } \\
\hline Never married & $1(4.0)$ & $2(8.7)$ \\
\hline Married & $17(68.0)$ & $17(73.9)$ \\
\hline Divorced & $5(20.0)$ & $1(4.4)$ \\
\hline Separated & $0(0.0)$ & $1(4.4)$ \\
\hline Widowed & $2(8.0)$ & $2(8.7)$ \\
\hline \multicolumn{3}{|l|}{ Employment (\%) } \\
\hline Currently working & $13(52.0)$ & $13(56.5)$ \\
\hline Worked in past 6 months & $2(8.0)$ & $1(4.4)$ \\
\hline Retired & $8(32.0)$ & $7(30.4)$ \\
\hline Not working $>6$ months & $2(8.0)$ & $2(8.7)$ \\
\hline \multicolumn{3}{|l|}{ Disease stage (AJCC 1997) (\%) } \\
\hline Stage 0 & $5(20.0)$ & $8(34.8)$ \\
\hline Stage I & $14(56.0)$ & $7(30.4)$ \\
\hline Stage II & $6(24.0)$ & $4(17.4)$ \\
\hline Stage III & $0(0.0)$ & $4(17.4)$ \\
\hline
\end{tabular}

randomized to the intervention, and 23 patients were randomized to standard care. The characteristics of the ITT groups are given in Table 1. It is noteworthy that the patient groups were similar with regard to gender, race (all Caucasian), age, years of education, marital status, employment history, and stage of melanoma (1997 AJCC staging system). Loss to follow-up over successive survey time points was noted for all groups. At the last survey time point (the 6-month follow-up), information was obtained from 18 of 25 patients (72\%) in the intervention group and 16 of 23 patients $(70 \%)$ in the standard care group.

In the EOI analysis, patients who chose not to attend the intervention were combined with individuals who were randomized to receive standard care, resulting in an intervention group ( $n=15$ patients) and a nonintervention group ( $n=33$ patients). The characteristics of the EOI groups are given in Table 2. Like the ITT analysis, the groups were similar with
TABLE 2

Patient Characteristics: Intervention Comparison

\begin{tabular}{|c|c|c|}
\hline Characteristic & $\begin{array}{l}\text { Randomized to } \\
\text { intervention }\end{array}$ & $\begin{array}{l}\text { Randomized to } \\
\text { control }\end{array}$ \\
\hline \multicolumn{3}{|l|}{ Sample size } \\
\hline Baseline & 15 & 33 \\
\hline Two months & 14 & 25 \\
\hline Six months & 13 & 21 \\
\hline \multicolumn{3}{|l|}{ Male gender } \\
\hline No. & 4 & 10 \\
\hline$\%$ at Month 2 & 26.7 & 30.3 \\
\hline \multicolumn{3}{|l|}{ Age (yrs) } \\
\hline Mean & 55.1 & 53.0 \\
\hline Range & $30-81$ & $22-92$ \\
\hline \multicolumn{3}{|l|}{ Education (yrs) } \\
\hline Mean & 15.3 & 14.5 \\
\hline Range & $12-23$ & $12-21$ \\
\hline \multicolumn{3}{|l|}{ No. of children } \\
\hline Median & 2.1 & 2.2 \\
\hline Range & $0-5$ & $0-5$ \\
\hline \multicolumn{3}{|l|}{ Marital status (\%) } \\
\hline Never married & $0(0.0)$ & $3(9.71)$ \\
\hline Married & $13(86.7)$ & $21(63.6)$ \\
\hline Divorced & $2(13.3)$ & $4(12.1)$ \\
\hline Separated & $0(0.0)$ & $1(3.0)$ \\
\hline Widowed & $0(0.0)$ & $4(12.1)$ \\
\hline \multicolumn{3}{|l|}{ Employment (\%) } \\
\hline Currently working & $7(46.7)$ & $19(57.6)$ \\
\hline Worked in past 6 months & $2(13.3)$ & $1(3.0)$ \\
\hline Retired & $4(26.7)$ & $11(33.3)$ \\
\hline Not working $>6$ months & $2(13.3)$ & $2(6.1)$ \\
\hline \multicolumn{3}{|l|}{ Disease stage (AJCC 1997) (\%) } \\
\hline Stage 0 & $4(26.7)$ & $9(27.3)$ \\
\hline Stage I & $9(60.0)$ & $12(36.4)$ \\
\hline Stage II & $2(13.3)$ & $8(24.2)$ \\
\hline Stage III & $0(0.0)$ & 4 (12.1) \\
\hline
\end{tabular}

regard to gender, race (all Caucasian), age, years of education, marital status, employment history, and stage of melanoma (1997 AJCC staging system). Loss to follow-up over successive survey time points was noted for all groups. At the last survey time point (the 6-month follow-up), information was obtained from 13 of 15 patients $(87 \%)$ who received the intervention and 21 of 33 patients $(64 \%)$ who did not receive the intervention.

\section{ITT Analyses}

At the time of randomization ( $\mathrm{t} 0$ ), groups had similar overall QOL, as measured by the SF-36 scales and the STAI Trait scale. In particular, groups were similar on the Physical Functioning, Bodily Pain, General Health, Social Functioning, and Mental Health scales of the SF-36. Table 3 reports the estimated mean scores for each scale at each time point from longitudinal mixedeffects models. 
TABLE 3

Quality-of-Life Intent-to-Treat Comparison: Estimated Means from Longitudinal Analyses by Randomization Group

\begin{tabular}{|c|c|c|c|c|}
\hline \multirow[b]{2}{*}{ Characteristic } & \multicolumn{3}{|c|}{ Mean (SE) } & \multirow[b]{2}{*}{$P$ value } \\
\hline & Randomized to intervention & Randomized to control & Contrast & \\
\hline \multicolumn{5}{|l|}{ Baseline } \\
\hline \multicolumn{5}{|l|}{ SF-36 } \\
\hline Physical functioning & $67.5(6.3)$ & $71.4(4.4)$ & $-3.9(7.7)$ & 0.62 \\
\hline Bodily pain & $64.1(4.3)$ & $55.4(5.5)$ & $8.7(7.0)$ & 0.22 \\
\hline General health & $65.8(4.1)$ & $57.7(4.8)$ & $8.1(6.3)$ & 0.21 \\
\hline Vitality & $47.9(4.0)$ & $40.0(4.5)$ & $7.9(6.0)$ & 0.20 \\
\hline Social functioning & $71.5(4.4)$ & $58.2(6.1)$ & $13.3(7.5)$ & 0.09 \\
\hline Mental health & $60.0(4.0)$ & $54.9(4.2)$ & $5.1(5.9)$ & 0.39 \\
\hline STAIT & $44.2(2.4)$ & $44.1(1.4)$ & $0.1(2.8)$ & 0.99 \\
\hline \multicolumn{5}{|l|}{ Two-month follow-up } \\
\hline \multicolumn{5}{|l|}{ SF-36 } \\
\hline Physical functioning & $76.4(5.9)$ & $78.7(4.0)$ & $-2.3(7.2)$ & 0.75 \\
\hline Bodily pain & $71.9(5.0)$ & $66.4(5.5)$ & $5.5(7.5)$ & 0.46 \\
\hline General health & $71.0(3.9)$ & $53.8(4.8)$ & $17.1(6.2)$ & 0.008 \\
\hline Vitality & $55.6(4.3)$ & $42.1(4.6)$ & $13.5(6.3)$ & 0.04 \\
\hline Social functioning & $85.9(4.8)$ & $70.1(5.7)$ & $15.8(7.4)$ & 0.04 \\
\hline Mental health & $72.6(3.9)$ & $57.0(4.7)$ & $15.6(6.1)$ & 0.01 \\
\hline STAIT & $39.3(2.4)$ & $44.4(2.2)$ & $-5.1(3.3)$ & 0.12 \\
\hline \multirow{2}{*}{\multicolumn{5}{|c|}{$\begin{array}{l}\text { Six-month follow-up } \\
\text { SF-36 }\end{array}$}} \\
\hline & & & & \\
\hline Physical functioning & $74.5(5.2)$ & $82.8(4.4)$ & $-8.3(6.8)$ & 0.23 \\
\hline Bodily pain & $70.1(5.6)$ & $70.2(4.8)$ & $-0.02(7.3)$ & 0.10 \\
\hline General health & $70.2(4.6)$ & $57.7(4.8)$ & $12.4(6.0)$ & 0.05 \\
\hline Vitality & $54.6(4.4)$ & $44.2(4.3)$ & $10.4(6.1)$ & 0.10 \\
\hline Social functioning & $86.1(4.7)$ & $79.4(4.3)$ & $6.6(6.4)$ & 0.30 \\
\hline Mental health & $72.3(3.5)$ & $61.5(5.0)$ & $10.8(6.1)$ & 0.09 \\
\hline STAIT & $38.5(2.2)$ & $45.4(2.8)$ & $-6.9(3.5)$ & 0.06 \\
\hline
\end{tabular}

Contrary to our hypothesis, distress, as measured by the GSI of the BSI, was not impacted significantly by intervention, although anxiety, as measured by the STAI State scale and the Anxiety scale of the BSI, were significantly lower for the intervention group at 2-month follow-up and 6-month follow-up, respectively. Table 4 presents the mean scores with standard errors and the significant differences between the two groups at each time point. Thus, although overall distress was not affected significantly by the intervention, significant reductions in anxiety were observed both immediately after the intervention and a short interval after the intervention.

Although the intervention was constructed to help patients reduce their level of distress, it also appears to have increased significantly several aspects of health functioning. In particular, General Health, Vitality, Social Functioning, and Mental Health scores of the SF-36 all improved immediately after treatment. When comparing patients who received the intervention with patients who received standard care, at the 2-month follow-up, General Health scores were 17.1 points higher $(P=0.008)$, Vitality scores were 13.5 points higher $(P=0.04)$, Social Functioning scores were 15.8 points higher $(P=0.04)$, and Mental Health scores were 15.6 points higher $(P=0.01)$ for the intervention group. Unfortunately, these differences were not observed at the 6-month follow-up, with the exception of General Health scores, which remained 12.4 points higher $(P<0.05)$ for the intervention group.

\section{EOI Analyses}

Like the ITT analyses, at the time of randomization (t0), groups had similar overall QOL, as measured by the SF-36 scales and the STAI Trait scale. The one exception to this was a significantly higher Vitality score in patients who received the intervention. Table 5 reports the estimated mean scores for each scale for each time point from longitudinal mixed-effects models.

Contrary to the results of the ITT analyses and consistent with our hypothesis, the GSI was impacted significantly by the intervention, an effect that also 
TABLE 4

Distress/Depression/Anxiety Intent-to-Treat Comparison: Estimated Means from Longitudinal Analyses by Randomization Group

\begin{tabular}{llrl}
\hline & & Mean (SE) & Contrast \\
\cline { 2 - 4 } Characteristic & Randomized to intervention & Randomized to control & \\
\hline Baseline & & & \\
$\quad$ BSI & & & $0.4(1.8)$ \\
$\quad$ Global severity index & $65.3(1.2)$ & $64.9(1.3)$ & $1.2(2.3)$ \\
$\quad$ Depression index & $64.9(1.3)$ & $63.6(1.9)$ & $-0.04(2.6)$ \\
$\quad$ Anxiety index & $62.4(1.6)$ & $62.5(2.0)$ & $-1.8(3.4)$ \\
STAIS & $44.3(2.2)$ & $46.1(2.6)$ & 0.81 \\
Two-month follow-up & & & 0.59 \\
BSI & $58.1(1.8)$ & $61.1(1.9)$ & $-3.0(2.6)$ \\
$\quad$ Global severity index & $59.5(1.9)$ & $60.3(2.2)$ & $-0.7(2.9)$ \\
$\quad$ Depression index & $54.7(1.8)$ & $59.3(2.2)$ & $-4.6(2.9)$ \\
Anxiety index & $37.1(2.3)$ & $45.6(2.9)$ & $-8.5(3.7)$ \\
$\quad$ STAIS & & & 0.69 \\
Six-month follow-up & & & 0.25 \\
BSI & $57.7(2.3)$ & $61.6(2.3)$ & 0.80 \\
$\quad$ Global severity index & $57.7(2.4)$ & $51.1(2.4)$ & $-4.0(3.2)$ \\
$\quad$ Depression index & $51.6(2.0)$ & $42.9(3.2)$ & $-3.4(3.4)$ \\
$\quad$ Anxiety index & $36.1(2.1)$ & & $-7.4(2.9)$ \\
STAIS & & $-6.8(4.1)$ \\
\hline a SE: standard error; BSI: Brief Symptom Inventory; STAIS: State-Trait Anxiety Inventory (State). & & 0.02 \\
\hline
\end{tabular}

was reflected in the STAI State score and the Anxiety scale of the BSI, at the 2-month follow-up. In each occasion, patients who received the intervention had lower scores, reflective of better emotional well being, compared with patients who did not receive the intervention. The 2-month differences were observed at the 6-month follow-up for the Anxiety scale, with differences in both the GSI and the STAI State scores approaching significance $(P=0.08$ and $P=0.05$, respectively). It also is noteworthy that the intervention group scores on the GSI at both 2-month followup and 6-month follow-up were in the normal range, whereas the scores for the nonintervention group were $>1$ standard deviation of the mean, indicating moderate distress. Table 6 presents the mean scores with standard errors and the significant differences between the two groups at each time point.

Comparison of patients who received the intervention with patients those who did not receive the intervention revealed significant improvements in Bodily Pain scores $(P>0.05)$ and General Health scores $(P=0.02)$ at the 2-month follow-up, with continued differences observed in Vitality scores $(P$ $=0.008$ ). For each scale, scores were higher, reflecting greater health functioning, for the intervention group. Vitality scores $(P=0.04)$ continued to be significantly higher for the intervention group at the 6-month follow-up, with General Health scores $(P=0.05)$ and Social Functioning scores $(P=0.07)$ approaching significance.

\section{DISCUSSION}

Increased levels of distress can have an adverse impact on an individual's ability to make decisions, adhere to treatment recommendations, or engage in posttreatment screening and preventive behaviors. ${ }^{32-35}$ Thus, identifying ways to reduce distress in individuals with cancer is of great importance. Previous studies designed to reduce distress in patients with cancer have reported positive improvements in distress, coping, and survival. ${ }^{9,19-22}$ The unstructured, lengthy, or group format of these interventions, however, may make them difficult to incorporate into existing medical systems. Because few brief, replicable therapeutic interventions aimed at decreasing distress and improving QOL have been tested, the current study was designed to determine whether a structured, individual intervention could reduce distress and improve QOL.

The primary objectives of the current study may be examined from an ITT approach and an EOI approach, because both methods answer separate yet equally important questions. The ITT approach examines the effectiveness of an intervention in changing a particular behavior or, in this study, reducing distress and, secondarily, improving QOL. Using this approach in the current study revealed that the intervention did not effectively reduce overall emotional distress, although it did reduce anxiety and improve several aspects of QOL. At the 2-month and 6-month follow-up 
TABLE 5

Quality-of-Life Intervention Comparison: Estimated Means from Longitudinal Analyses by Receipt of Intervention

\begin{tabular}{|c|c|c|c|c|}
\hline \multirow[b]{2}{*}{ Characteristic } & \multicolumn{3}{|c|}{ Mean (SE) } & \multirow[b]{2}{*}{$P$ value } \\
\hline & Received intervention & Did not receive intervention & Contrast & \\
\hline \multicolumn{5}{|l|}{ Baseline } \\
\hline \multicolumn{5}{|l|}{ SF-36 } \\
\hline Physical functioning & $72.9(6.6)$ & $67.8(4.6)$ & $5.1(8.0)$ & 0.53 \\
\hline Bodily pain & $66.5(4.1)$ & $57.0(4.6)$ & $9.5(6.2)$ & 0.14 \\
\hline General health & $65.2(5.1)$ & $60.4(4.0)$ & $4.7(6.5)$ & 0.47 \\
\hline Vitality & $54.6(4.5)$ & $39.6(3.6)$ & $14.9(5.8)$ & 0.01 \\
\hline Social functioning & $71.2(5.5)$ & $62.3(5.0)$ & $8.9(7.4)$ & 0.24 \\
\hline Mental health & $57.9(5.5)$ & $57.3(3.5)$ & $0.6(6.5)$ & 0.93 \\
\hline STAIT & $44.6(3.4)$ & $44.0(1.4)$ & $0.7(3.6)$ & 0.85 \\
\hline \multicolumn{5}{|l|}{ Two-month follow-up } \\
\hline \multicolumn{5}{|l|}{ SF-36 } \\
\hline Physical functioning & $83.6(6.1)$ & $74.3(4.6)$ & $9.3(7.6)$ & 0.23 \\
\hline Bodily pain & $78.7(5.2)$ & $64.4(4.7)$ & $14.4(7.0)$ & 0.05 \\
\hline General health & $73.3(4.8)$ & $57.7(4.0)$ & $15.6(6.2)$ & 0.02 \\
\hline Vitality & $60.9(5.2)$ & $43.3(3.7)$ & $17.6(6.4)$ & 0.008 \\
\hline Social functioning & $85.6(5.2)$ & $74.8(4.9)$ & $10.8(7.1)$ & 0.14 \\
\hline Mental health & $72.5(5.3)$ & $61.6(3.8)$ & $10.9(6.5)$ & 0.10 \\
\hline STAIT & $39.3(3.2)$ & $43.0(1.9)$ & $-3.7(3.7)$ & 0.33 \\
\hline \multirow{2}{*}{\multicolumn{5}{|c|}{$\begin{array}{l}\text { Six-month follow-up } \\
\text { SF-36 }\end{array}$}} \\
\hline & & & & \\
\hline Physical functioning & $79.3(5.0)$ & $77.7(4.8)$ & $1.6(6.9)$ & 0.82 \\
\hline Bodily pain & $75.5(6.0)$ & $68.8(4.3)$ & $6.8(7.4)$ & 0.37 \\
\hline General health & $72.0(4.5)$ & $60.0(3.9)$ & $12.0(6.0)$ & 0.05 \\
\hline Vitality & $58.7(5.8)$ & $44.7(3.4)$ & $14.0(6.7)$ & 0.04 \\
\hline Social functioning & $89.9(4.1)$ & $78.9(4.2)$ & $11.0(5.9)$ & 0.07 \\
\hline Mental health & $72.0(4.8)$ & $64.6(3.9)$ & $7.4(6.2)$ & 0.24 \\
\hline STAIT & $37.9(2.9)$ & $43.7(2.3)$ & $-5.7(3.6)$ & 0.12 \\
\hline
\end{tabular}

assessments, anxiety was significantly lower in the intervention group compared with the standard care group, suggesting that improvements can be achieved through a brief, portable, easily administered, manualized CBI. In support of our secondary objectives, an additional positive outcome was improvement in QOL, as measured by the General Health, Vitality, Social Functioning, and Mental Health scales of the SF-36. Initial benefits (2-month assessment) of the intervention were most obvious, whereas the 6-month follow-up analyses revealed that many of the gains made at the initial assessment were reduced over time, although the intervention group continued to report greater levels of General Health compared with the standard care group.

Several issues arise in response to the observed findings, including the need to determine why the intervention was not effective in reducing general distress. The lack of effectiveness may have been the result of an inadequate number of sessions or provision of material in the intervention that did not have a direct effect on distress. Although it is possible that four sessions were not sufficient for changing an individual's level of distress, other studies have found improvements after similar numbers of sessions and even with fewer sessions. ${ }^{18,21}$ In addition, the material used in the sessions has been used in prior studies with demonstrated effectiveness. ${ }^{21,22}$ Therefore, it is likely that another issue was responsible for the lack of findings. An examination of the responses of individuals who were randomized to the intervention revealed that almost half of the intervention group declined to receive the intervention. Because acceptance of a treatment is an important part of determining whether a treatment program is effective, this finding may account for the lack of a significant difference between groups. In the ITT analysis, patients who declined to receive the intervention were included among the patients who received it, a practice, it may be argued, that reduces the likelihood of finding significant differences and does not provide an accurate description of the ability of the intervention to reduce distress, despite identifying problems with the acceptance of the intervention. Examination of the reasons 
TABLE 6

Distress/Depression/Anxiety Intervention Comparison: Estimated Means from Longitudinal Analyses by Receipt of Intervention

\begin{tabular}{|c|c|c|c|c|}
\hline \multirow[b]{2}{*}{ Characteristic } & \multicolumn{3}{|c|}{ Mean (SE) } & \multirow[b]{2}{*}{$P$ value } \\
\hline & Received intervention & Did not receive intervention & Contrast & \\
\hline \multicolumn{5}{|l|}{ Baseline } \\
\hline \multicolumn{5}{|l|}{ BSI } \\
\hline Global severity index & $65.9(1.8)$ & $64.7(1.0)$ & $1.2(2.1)$ & 0.57 \\
\hline Depression index & $65.7(1.9)$ & $63.5(1.4)$ & $2.2(2.4)$ & 0.37 \\
\hline Anxiety index & $63.7(2.3)$ & $61.9(1.5)$ & $1.9(2.8)$ & 0.50 \\
\hline STAIS & $45.8(3.1)$ & $45.0(2.0)$ & $0.8(3.7)$ & 0.83 \\
\hline \multicolumn{5}{|l|}{ Two-month follow-up } \\
\hline \multicolumn{5}{|l|}{ BSI } \\
\hline Global severity index & $55.1(2.3)$ & $62.1(1.5)$ & $-7.0(2.8)$ & 0.01 \\
\hline Depression index & $56.8(2.4)$ & $61.7(1.8)$ & $-4.9(3.0)$ & 0.11 \\
\hline Anxiety index & $51.4(2.5)$ & $59.6(1.7)$ & $-8.2(3.0)$ & 0.009 \\
\hline STAIS & $35.5(3.1)$ & $43.9(2.3)$ & $-8.3(3.8)$ & 0.03 \\
\hline \multicolumn{5}{|l|}{ Six-month follow-up } \\
\hline \multicolumn{5}{|l|}{ BSI } \\
\hline Global severity index & $55.4(2.7)$ & $61.5(1.9)$ & $-6.1(3.3)$ & 0.083 \\
\hline Depression index & $56.3(2.8)$ & $60.8(2.1)$ & $-4.5(3.5)$ & 0.20 \\
\hline Anxiety index & $49.3(2.5)$ & $58.2(1.8)$ & $-8.9(3.0)$ & 0.01 \\
\hline STAIS & $34.5(2.7)$ & $42.1(2.7)$ & $-7.6(3.8)$ & 0.05 \\
\hline
\end{tabular}

why individuals declined to receive the intervention included having to travel back to the medical center, living too far away, and not believing that the intervention was needed. Therefore, it appears that the reasons for the lack of acceptance were not inherent in the intervention but in practical issues with transportation and distance. This finding points to the need to identify additional intervention strategies that would overcome these limitations.

The identification of patients who declined to engage in the intervention led us to use a second EOI analysis to determine the efficacy of the intervention. In that analysis, the intervention did have the intended effect of reducing distress, with 2-month GSI scores in the intervention group that were significantly lower compared with the nonintervention group. Levels of anxiety also were lower in the intervention group at the 2-month follow-up. The importance of these results is tempered somewhat by the finding that at the 6-month follow-up, the differences between groups in terms of overall distress only approached significance. Despite the lack of a statistical difference, the intervention group continued to have BSI scores in the normal range, whereas the nonintervention group continued to exceed the level that has been identified as indicative of moderate distress. ${ }^{7,23,24}$ In addition, patients in the intervention group continued to report improved health-related QOL.

The lack of longer-term improvements in the pa- tients who received the intervention is not surprising in light of the fact that patients received only one session on each topic designed to reduce distress. Traditional cognitive-behavioral therapy is designed to produce change in thoughts and behaviors after repeated presentation of the material and exercises for patient integration of the strategies learned. For example, the problem-solving therapy that was presented in 1 session in the current study has been presented and revisited repeatedly over 10 sessions of therapy. ${ }^{36}$ This suggests that a longer intervention, which would provide multiple sessions for patient learning as well as effective implementation of these strategies, would result in longer-term change. Because such an approach may be too intensive for clinic settings, repeated bolus maintenance sessions provided on a regular basis may be beneficial in extending the initial change observed in the current study. Nevertheless, the results from the current study provide initial support for identifying individuals at increased distress, providing them with an intervention, and tracking their progress.

We advocated this approach in a previous report ${ }^{37}$ and believe that such an approach should be considered when attempting replication in either melanoma or other oncology samples. This approach would include 1) targeting a clinic with a high enough clinical output that $30 \%$ of patients (those likely to be in distress) would represent enough participants to test an 
intervention; 2) triaging only those patients most likely to benefit from the intervention (i.e., moderately-tohighly distressed patients) to treatment; 3) treating patients using methods likely to be associated with minimal disruption of medical clinical operations but with maximum reliability and power for measuring change, such as CBIs (e.g., relaxation training, cognitive reframing, information on coping) that have proven efficacy in patients with cancer and other health conditions ${ }^{18-21,38,39}$; and 4) tracking participant change using multiple outcome measures (e.g., QOL, distress, coping, adherence, resource utilization) to further refine the first 3 steps.

Tracking in the current study revealed that patients did not complete assessments at all occasions. In fact, approximately $30 \%$ of the sample did not complete 6-month questionnaires. The loss of patients to follow-up is a limitation in the current study that resulted in the use of a mixed-effects model analysis to average patient scores instead of a repeated-measures analysis of variance. This method was chosen to use each patient's response where it occurred and to allow the inclusion patients in the model with the data they completed. Despite the loss of data, the drop-out pattern was the same for each group, suggesting that if recurrences or other disease-related issues were the reasons for dropping out, then they affected both groups equally. Nevertheless, the loss of patient data over time indicates the need for increased attention to and implementation of mechanisms in future longitudinal studies designed to increase questionnaire completion.

In conclusion, the current study reports on the provision of a short-term CBI for reducing distress and improving QOL in a sample of patients with melanoma who had high-to-medium distress. Although increased attention and further research are needed to develop ways of increasing the acceptance of the intervention, the results provide initial support for its efficacy in the short term and the ability to maintain lower levels of distress over a 6-month period. Continued research using a 4-T approach, as discussed above, will be important in reducing the distress that is present in a significant number of patients who are confronted with new diagnoses of cancer and discussions of its treatments.

\section{REFERENCES}

1. Jackson A, Wilkinson C, Ranger M, Pill R, August P. General practice: can primary prevention or selective screening for melanoma be more precisely targeted through general practice? A prospective study to validate a self-administered risk score. Br Med J. 1998;316:34-38.

2. Marks R. Epidemiology of melanoma. Clin Exp Dermatol. 2000;25:459-463.
3. National Cancer Institute. Surveillance, epidemiology, and end results [monograph online]. Available from URL: http:// www.seer.cancer.gov [Accessed February 2003]

4. American Cancer Society. Cancer facts and figures, 2002. Atlanta: American Cancer Society, 2002.

5. Balch CM, Buzaid AC, Seng-Jaw S, et al. Final version of the American Joint Committee on Cancer staging system for cutaneous melanoma. J Clin Oncol. 2001;19:3635-3648.

6. Trask PC, Paterson AG, Hayasaka S, Dunn RL, Riba M, Johnson T. Psychosocial characteristics of individuals with non-Stage IV melanoma. J Clin Oncol. 2001;19:2844-2850.

7. Derogatis LP, Morrow GR, Fetting J, et al. The prevalence of psychiatric disorders among cancer patients. JAMA. 1983; 249:751-757.

8. Sollner W, Zingg-Schir M, Rumpold G, Mairinger G, Fritsch $P$. Need for supportive counseling-the professionals' versus the patients' perspective. Psychother Psychosom. 1998;67: 94-104.

9. Fawzy FI, Fawzy NW, Hyun CS, et al. Malignant melanoma. Effects of an early structured psychiatric intervention, coping, and affective state on recurrence and survival 6 years later. Arch Gen Psychiatry. 1993;50:681-689.

10. Havlik RJ, Vukasin AP, Ariyan S. The impact of stress on the clinical presentation of melanoma. Plast Reconstr Surg. 1992;90:57-64.

11. DiMatteo MR, Lepper HS, Croghan TW. Depression is a risk factor for noncompliance with medical treatment. Arch Intern Med. 2000;160:2101-2107.

12. Batel-Copel LM, Kornblith AB, Batel PC, Holland JC. Do oncologists have an increasing interest in the quality of life of their patients? A literature review of the last 15 years. Eur J Cancer. 1997;1:29-32.

13. Cella DF, Bonomi AE. Measuring quality of life: 1995 update. Oncology. 1995;9:47-60.

14. Portenoy RK, Thaler HT, Kornblith AB, et al. Symptom prevalence, characteristics and distress in a cancer population. Qual Life Res. 1994;3:183-189.

15. Schipper H, Clinch J, McMurray M, Levitt M. Measuring the quality of life of cancer patients: the Functional Living Index-Cancer: development and validation. J Clin Oncol. 1984; 5:472-473.

16. Brown JE, King MT, Butow PN, Dunn SM, Coates AS. Patterns over time in quality of life, coping and psychological adjustment in late stage melanoma patients. Qual Life Res. 2000;9:75-85.

17. Trijsburg RW, van Knippenberg FCE, Rijpma SE. Effects of psychological treatment on cancer patients: a critical review. Psychosom Med. 1992;54:489-517.

18. Andersen BL. Biobehavioral outcomes following psychological interventions for cancer patients. J Consult Clin Psychol. 2002;70:590-610.

19. Alter CL, Fleishman S, Kornblith A, et al. Supportive telephone intervention for patients receiving chemotherapy: a pilot study. Psychosomatics. 1996;37:425-431.

20. Donnelly J, Kornblith AB, Fleishman S, et al. A pilot study of interpersonal psychotherapy by telephone with cancer patients and their partners. Psychooncology. 2000;9:44-56.

21. Fawzy FI. Psychosocial interventions for patients with cancer: what works and what doesn't. Eur J Cancer. 1999;35: $1559-1564$.

22. Fawzy FI, Fawzy NW, Arndt LA, Pasnau RO. Critical review of psychosocial interventions in cancer care. Arch Gen Psychiatry. 1995;52:100-113. 
23. Derogatis LR. The Brief Symptom Inventory (BSI): administration, scoring and procedures. Manual II. Baltimore: Clinical Psychometric Research, Inc., 1992.

24. Derogatis LR, Melisaratos N. The Brief Symptom Inventory: an introductory report. Psychol Med. 1983;13:595-605.

25. Boulet J, Boss MW. Reliability and validity of the Brief Symptom Inventory. Psychol Assess. 1991;3:433-437.

26. Ware JE. SF-36 Health Survey: manual and interpretation guide. Boston: Health Institute, New England Medical Center, 1993.

27. Spielberger CD. Manual for the State-Trait Anxiety Inventory: STAI (Form Y). Palo Alto: Consulting Psychologists Press, 1983.

28. Spielberger CD, Sydeman SJ. State-Trait Anxiety Inventory and State-Trait Anger Expression Inventory. In: Maruish ME, editor. The use of psychological tests for treatment planning and outcome assessment. Hillsdale, NJ: Lawrence Erlbaum Associates, 1994:292-321.

29. Verbeke G, Molenberghs G. Linear mixed models in practice: a SAS-oriented approach. New York: Springer-Verlag, 1997.

30. Laird NM, Ware JH. Random effects models for longitudinal data. Biometrics. 1982;38:963-974.

31. Laird NM. Missing data in longitudinal studies. Stat Med. 1988;7:305-315.

32. Dermatis H, Lesko LM. Psychological distress in parents consenting to child's bone marrow transplantation. Bone Marrow Transplant. 1990;6:411-417.
33. Dermatis H, Lesko LM. Psychosocial correlates of physicianpatient communication at time of informed consent for bone marrow transplantation. Cancer Invest. 1991;9:621628.

34. Raghunathan R, Pham MT. All negative moods are not equal: motivational influences of anxiety and sadness on decision-making. Organ Behav Hum Decis Process. 1999;79: 56-77.

35. Glasgow RE, Orleans CT. Adherence to smoking cessation regimens. In: Gochman DS, editor. Handbook of health behavior research. Volume 2. Provider determinants. New York: Plenum Press, 1997:353-377.

36. Nezu AM, Nezu CM, Friedman SH, Faddis S, Houts PS. Helping cancer patients cope: a problem-solving approach. Washington: American Psychological Association, 1998.

37. Trask PC, Schwartz SM, Deaner SL, et al. Behavioral medicine: the challenge of integrating psychological and behavioral approaches into primary care. Eff Clin Pract. 2002;5: 75-83.

38. Andersson G, Stromgren T, Strom L, Lyttkens L. Randomized controlled trial of internet-based cognitive behavior therapy for distress associated with tinnitus. Psychosom Med. 2002;64:810-816.

39. Strom L, Pettersson R, Andersson G. A controlled trial of self-help treatment of recurrent headache conducted via the Internet. J Consult Clin Psychol. 2000;68:722-727. 